\title{
Searching for minor absorptions on D-type asteroids
}

\author{
Thais Mothé-Diniz \\ Universidade Federal do Rio de Janeiro, Observatório do Valongo \\ Rio de Janeiro, RJ, Brazil \\ email: thais.mothe@astro.ufrj.br
}

\begin{abstract}
Preferably located in the outer main belt, D-type asteroids experienced less heating and represent an important population for studies on the origin and evolution of the asteroid belt, as well as the relations between asteroidal and cometary bodies. Their surface mineralogy is currently related to a mixture of organics, anhydrous silicates, opaque material and ice. However, like other taxonomic classes, a large spectral diversity can be seen among D-type objects. We use the Visible spectra of 100 D-type objects available in the literature to search for minor absorptions in those objects. The presence of minor absorptions around 0.6 and 0.8 microns is reported for a large number of objects in the sample. The presence of such bands is not related to the heliocentric distance of the objects, since the absorptions can be seen in the whole main belt, up to the the Trojans region.
\end{abstract}

Keywords. Asteroids, D-types, absorptions

\section{Introduction}

D-type asteroids probably experienced less heating and represent important relics from early epochs of Solar System formation. Their study may help to better understand the origin and evolution of the asteroid belt, as well as the relations between asteroidal and cometary bodies. Inferring the composition of D-type asteroids is specially difficult since, apart from the recently discovered meteorites Tagish Lake and WIS91600, no other meteorite analogue has been found on Earth. From their spectral slope and apparent absence of features, the surface mineralogy of D-type asteroids is currently related to a mixture of organics, anhydrous silicates, opaque material and ice. These objects dominate the external region (from $\sim 3.2 \mathrm{AU}$ ) of the main asteroid belt, being abundant among the Cybeles (between 3.3 and 3.5 AU), the Hildas (around 4.0 AU, in the 3:2 resonance with Jupiter) and the Trojans (at 5.2 AU, around the Lagrangian points $\mathrm{L}_{4}$ and $\mathrm{L}_{5}$ of Jupiter). However, a non-negligible amount of these objects can also be found in the inner regions of the belt, from about 2.1 AU.

Previous spectroscopic studies of D-types have been preferably done in the ambit of the study of dynamical associations. The Hilda and Cybele asteroids were investigated by Dahlgren et al. (1997) and Lagerkvist et al. (2005). The Trojans by Jewitt \& Luu(1990), Emery \& Brown (2003), Fornasier et al. (2004), Dotto et al. (2005). Other studies of lowalbedo asteroids were performed by Vilas \& McFadden(1992), Vilas et al. (1993), Dumas et al. (1998). Besides that, some D-type asteroids have been associated to low-activity comets through dynamical, physical and spectroscopical constraints (Campins et al. 1987; Millis et al. 1988; Luu 1993; Harris et al. 2001; Abell et al. 2005; Campins et al. 2005).

Investigations by Jones et al. (1990) in the region around $3 \mu \mathrm{m}$ and by Vilas et al. (1994) in the visible, suggested that these objects lack hydrated minerals in their surfaces. 
Cruikshank et al. (2001), on the other hand, proposed that if the surfaces of the D-type asteroids are rich in opaque phases, they may contain significant amounts of hydrosilicates. The authors, however, did not show any detectable absorption band in the visible and near-infrared (NIR) in that work. Indeed, recently Kanno et al. (2003) reported the first detection of a $3 \mu \mathrm{m}$ band in a D-type asteroid: (773) Irmintraud. They also showed that the shape of the band on (773) was very similar to that on the asteroid (1) Ceres.

Differences among D-type asteroids have been investigated by several authors, like Lagerkvist et al. (1993) who reported a clear correlation between the color index and the heliocentric distance, and Carvano et al. (2003) who noticed that inner main-belt $\mathrm{D}$-types have more concave spectral shapes and higher albedos than those in the outer main-belt. In this work we study the spectra of $100 \mathrm{D}$-type asteroids, obtained from the public spectroscopic surveys SMASSII (Bus \& Binzel 2002), S3OS2 (Lazzaro et al. 2004), and from the work of Fornasier et al. (2004), where it is possible to find the details of the respective observations. The data are composed of low resolution spectra on the $0.44 / 0.5$ $-0.92 \mu \mathrm{m}$ range, all classified according to the Bus taxonomic system (Bus \& Binzel 2002). Although belonging to the Centaurs group, the object (10199) Chariklo observed by the S3OS2 survey was included in the search for absorptions, since its spectral slope is similar to that of some trojan D-types. The relevant orbital and physical parameters for all objects in this study can be seen in Table 1 .

Our first step was to search for correlations among physical and orbital parameters, including the Tisserand parameter $\mathrm{T}_{J}$. Subsequently, a search for small absorption bands was performed, and we examined the possible correlations between the presence/absence of bands with all the parameters available. The results of these searches are presented in the sections that follow.

\section{Search for absorptions and correlations on D-type asteroids}

Search for minor features. In the search for minor absorption bands, we followed the approach of Vilas et al. (1993) to enhance minor spectral features present in the spectra. We have first smoothed each spectrum with a running-box average in order to reduce the spectral resolution, and then divided by a linear continuum across the spectral interval of $0.5-0.92 \mu \mathrm{m}$. With this procedure, we have detected subtle features near $0.60-0.65$ $\mu \mathrm{m}$ and $0.80-0.9 \mu \mathrm{m}$, with depths down to about $2 \%$ of the background continuum, on many objects in our sample. These features are illustrated in Fig. 1. A confidence code was assigned according to the noise level of the spectra and repeatability of the feature between different spectra of the same asteroid. Whenever an absorption band deeper than our detection limit was found in one or more spectra of an object after the continuum removal, then it was considered a "positive" identification. Many spectra presented features that were not well defined, with depths smaller than the detection limits and that were absent in other spectra from different nights/observing times. In this case, we considered the feature as "doubtful". The detection limits are determined from the $\mathrm{S} / \mathrm{N}$ of the spectra in the region of the bands, which are, in average, between 1-3\%. The "positive" identifications are represented in Table 1 with a cross (x). Objects presenting a doubtful feature in either or both regions are: 267, 612, 666, 717, 729, 732 , 891, 1361, 1689, 2105, 2246, 2263, 2569, 2872, 3063, 3248, 3709, 3793, 4063, 4068, 4103, 4489, 4617, 4744, 4833, 4835, 5264, 5461, 5818, 6545, 7516, 8513, 13463, and 30698 . Objects showing no absorption at all are: 361, 520, 565, 911, 1094, 1118, 1226, 1293, 1328, 1535, 2086, 2454, 2677, 2891, 3015, 3141, 3333, 3990, 4035, 4902, 5258, 5362, 5914, 7635, 9430, 11351, 12917, 14465, 15502, 20738, and 24390. Object (818) Kapytenia presents 
Table 1. Main physical parameters of the asteroids studied. The positive identification of the 0.6 and/or $0.8 \mu \mathrm{m}$ bands is reported with a "x", while a "?" denotes a doubtful feature. Any dynamical association is also listed.

\begin{tabular}{|c|c|c|c|c|c|c|c|c|c|c|}
\hline Number & Name & $\mathrm{a}$ & $\mathrm{p}_{\nu}$ & $\mathrm{D}(\mathrm{km})$ & $\mathrm{T}_{J}$ & Dyn.Assoc. & Slope & Concavity & $0.6 ?$ & $0.8 ?$ \\
\hline 12 & Victoria & 2.34 & $0.176^{*}$ & 112.77 & 3.52 & & 10.1 & $\mathrm{ccv}$ & $\mathrm{x}$ & $\mathrm{x}$ \\
\hline 510 & Mabella & 2.61 & 0.07 & 57.40 & 3.36 & - & 7.0 & $\mathrm{ccv}$ & $\mathrm{x}$ & - \\
\hline 547 & Praxedis & 2.77 & 0.06 & 69.60 & 3.23 & - & 6.3 & $\mathrm{ccv}$ & $\mathrm{x}$ & - \\
\hline 579 & Sidonia & 3.01 & 0.175 & 85.56 & 3.22 & Eos & 7.9 & $\mathrm{ccv}$ & $\mathrm{x}$ & $\mathrm{x}$ \\
\hline 721 & Tabora & 3.55 & 0.060 & 76.07 & 3.09 & Cybele & 8.3 & flat & $\mathrm{x}$ & $\mathrm{x}$ \\
\hline 726 & Joella & 2.56 & 0.05 & 44.00 & 3.33 & - & 6.3 & flat & $\mathrm{x}$ & $\mathrm{x}$ \\
\hline 775 & Lumiere & 3.01 & 0.108 & 33.59 & 3.23 & Eos & 8.8 & $\mathrm{ccv}$ & - & $\mathrm{x}$ \\
\hline 798 & Ruth & 3.01 & 0.16 & 43.20 & 3.23 & Eos & 6.4 & $\mathrm{ccv}$ & $\mathrm{x}$ & $\mathrm{x}$ \\
\hline 818 & Kapteynia & 3.17 & 0.165 & 49.45 & 3.14 & & 8.6 & $\mathrm{ccV}$ & $?$ & $\mathrm{x}$ \\
\hline 1006 & Lagrangea & 3.15 & 0.07 & 29.60 & 3.08 & - & 6.7 & flat & $\mathrm{x}$ & - \\
\hline 1172 & Aneas & 5.19 & - & 142.80 & 2.91 & Trojan* & 9.1 & flat & $\mathrm{x}$ & $\mathrm{x}$ \\
\hline 1209 & Pumma & 3.17 & - & - & 3.18 & - & 6.9 & $\mathrm{ccv}$ & $\mathrm{x}$ & - \\
\hline 1275 & Cimbria & 2.68 & 0.11 & 29.20 & 3.32 & Eunomia & 6.4 & cvx & - & $\mathrm{x}$ \\
\hline 1284 & Latvia & 2.65 & 0.104 & 36.81 & 3.35 & & 8.5 & $\mathrm{ccv}$ & $\mathrm{x}$ & $\mathrm{x}$ \\
\hline 1321 & Majuba & 2.94 & 0.143 & 36.30 & 3.23 & & 8.3 & $\mathrm{ccv}$ & - & $\mathrm{x}$ \\
\hline 1328 & Devota & 3.51 & 0.04 & 57.20 & 3.10 & - & 14.5 & flat & - & - \\
\hline 1400 & Tirela & 3.12 & - & - & 3.12 & Tirela & 8.2 & $\mathrm{ccv}$ & $\mathrm{x}$ & $\mathrm{x}$ \\
\hline 1481 & Tubingia & 3.01 & 0.117 & 33.26 & 3.24 & & 7.8 & $\mathrm{ccv}$ & $\mathrm{x}$ & - \\
\hline 1542 & Schalen & 3.10 & 0.065 & 45.19 & 3.21 & & 9.0 & $\mathrm{ccv}$ & $\mathrm{x}$ & $\mathrm{x}$ \\
\hline 1574 & Meyer & 3.54 & 0.039 & 58.68 & 3.07 & Cybele & 10.2 & flat & $\mathrm{x}$ & - \\
\hline 1609 & Brenda & 2.58 & 0.115 & 29.64 & 3.31 & & 9.8 & $\mathrm{ccV}$ & $\mathrm{x}$ & $\mathrm{x}$ \\
\hline 1647 & Menelaus & 5.24 & - & - & 2.99 & Trojan* & 6.7 & flat & $\mathrm{x}$ & - \\
\hline 2235 & Vittore & 3.22 & 0.047 & 44.45 & 3.07 & & 10.1 & flat & - & $\mathrm{x}$ \\
\hline 2266 & Tchaikovsky & 3.39 & 0.038 & 46.94 & 3.08 & Cybele & 10.0 & flat & $\mathrm{x}$ & $\mathrm{x}$ \\
\hline 2448 & Sholokhov & 2.79 & 0.13 & 30.20 & 3.25 & - & 8.3 & flat & $\mathrm{x}$ & - \\
\hline 2498 & Tsesevich & 2.92 & - & - & 3.28 & Koronis & 7.3 & $\mathrm{ccv}$ & $\mathrm{x}$ & $\mathrm{x}$ \\
\hline 2867 & Steins & 2.36 & - & - & 3.51 & Inner & 7.0 & $\mathrm{ccv}$ & - & $\mathrm{x}$ \\
\hline 2959 & Scholl & 3.95 & 0.05 & 34.20 & 2.99 & - & 14.2 & flat & $\mathrm{x}$ & - \\
\hline 3140 & Stellafane & 3.02 & 0.126 & 24.75 & 3.21 & Eos & 8.2 & flat & $\mathrm{x}$ & $\mathrm{x}$ \\
\hline 3152 & Jones & 2.63 & 0.05 & 33.20 & 3.37 & - & 8.3 & flat & $\mathrm{x}$ & $\mathrm{x}$ \\
\hline 3453 & Dostoevsky & 2.39 & - & - & 3.53 & & 7.3 & flat & $\mathrm{x}$ & $\mathrm{x}$ \\
\hline 3682 & Welther & 2.75 & 0.12 & 19.40 & 3.23 & - & 6.2 & $\mathrm{ccv}$ & $\mathrm{x}$ & - \\
\hline 3906 & Chao & 2.93 & 0.04 & 47.00 & 3.12 & - & 5.2 & flat & $\mathrm{x}$ & - \\
\hline 5648 & 1990VU1 & 5.17 & - & - & 2.82 & - & 12.8 & flat & $\mathrm{x}$ & - \\
\hline 10199 & Chariklo & 15.79 & - & - & 3.48 & Centaur & 10.3 & flat & $\mathrm{x}$ & $\mathrm{x}$ \\
\hline 15535 & 2000AT177 & 5.16 & - & - & 2.94 & Trojan* & 11.7 & flat & $\mathrm{x}$ & $\mathrm{x}$ \\
\hline
\end{tabular}

one positive identification around $0.8 \mu \mathrm{m}$ and one doubtful identification around $0.6 \mu \mathrm{m}$. The later is denoted on Table 1 as a "?".

Considering only the positive identifications, $\approx 12 \%$ of our sample presents only the 0.6-0.65 $\mu \mathrm{m}$ band, while $5 \%$ only the $0.8-0.9 \mu \mathrm{m}$ band and $\approx 18 \%$ of the sample has both bands. If we take into account also the doubtful features, these percentages raise to $21 \%$, $12 \%$ and $32 \%$ respectively. Inspection of the relations between the presence of features and orbital and physical parameters reveals that unambiguous positive identifications were found in the whole main-belt and Trojan population.

Testing for correlations. Correlations were searched among the parameters using the Spearman rank order correlation. The following orbital and physical parameters were used: semi-major axis $a$, absolute magnitude $H$, albedo $\rho_{\nu}$, diameter $D$ and slope $S^{\prime}$. The spectra were normalized to unity at $0.6 \mu \mathrm{m}$, and a linear reflectance slope $S^{\prime}$ of each 

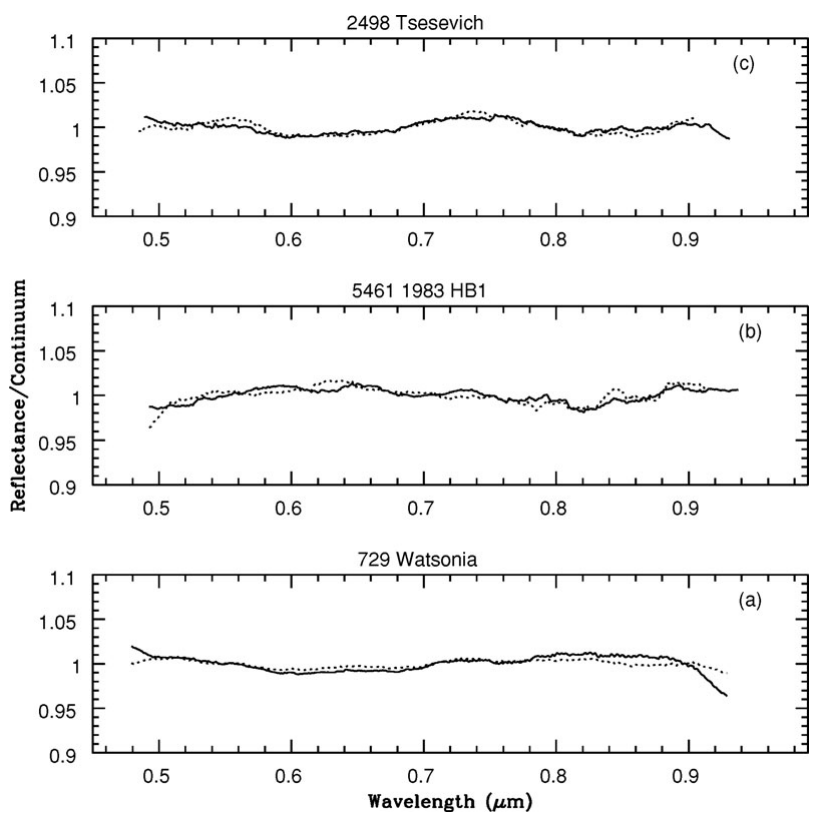

Figure 1. Reflectance spectra of three D-type asteroids smoothed with a running-box average. The spectra have also been divided by a 2-degree polynomial fit, in order to remove the continuum. Different line types denote spectra from different nights/observing runs. (a) spectrum with a $0.6 \mu m$ band, (b) with a $0.8 \mu m$ band and (c) with both the 0.6 and $0.8 \mu m$ bands.

spectrum was defined as the angular coefficient of a linear fit to the spectrum in the 0.50-92 $\mu \mathrm{m}$ range, given in $\% 10^{3} \AA$. The morphology of the spectra was also inspected, and each object was classified in one of the three groups: concave (ccv) $\cap$, convex (cvx) $\cup$ or flat /. The curvatures for the spectra presenting features are shown on Table 1 . Within a confidence level of $99 \%$ and using the entire sample, a correlation was found between the following pairs of parameters: $\rho_{\nu}$ and $T_{J}, \mathrm{H}$ and $T_{J}$. Anti-correlation is seen between $\rho_{\nu}$ and $D, \rho_{\nu}$ and the semi-major axis $a$, and between $\mathrm{D}$ and $T_{J}$. At a confidence level of $95 \%$ an anti-correlation between $T_{J}$ and $S^{\prime}$ is also reported, as well as a correlation between $a$ and $S^{\prime}$. It is important to notice that Carvano et al. (2003) remark that the correlation between $a$ and $S^{\prime}$ depends on the normalization wavelength, and therefore, cannot be considered significant. Only one "cvx" spectrum in our sample presents absorptions, and we report a predominance of "flat" spectra with these features.

\section{Discussion}

Absorption features centered near 0.6-0.65 $\mu \mathrm{m}$ and 0.8-0.9 $\mu \mathrm{m}$ have been detected for the first time in a large number of D-type asteroids. Previously, Vilas et al. (1993) reported both bands in a small number of asteroids: 165, 225, 334 ,368, and 877, which are all low-albedo and not steeply sloped. They attributed those bands respectively to $6 \mathrm{~A} 1 \rightarrow 4 \mathrm{~T} 2(\mathrm{G})$ and $6 \mathrm{~A} 1 \rightarrow 4 \mathrm{~T} 1(\mathrm{G})$ charge transfer transitions $(\mathrm{Fe} 3+\rightarrow \mathrm{Fe})$ in minerals such as the oxyhydroxide goethite, the $\mathrm{Fe}$ oxide hematite and the sulfate jarosite. They are products of the aqueous alteration of anhydrous silicates. Analogues for these features are seen in spectra of CM2 carbonaceous chondrites Cold Bokkeveld and Murray. 
In this work the literature (eg. Burns 1993) was searched for alternatives for both features. The only mineral found with both absorptions seem to be the Andradite, which is a calcium-iron silicate, where they occur via crystal field transitions. This mineral is found on the CV3 Chondrite Allende. Recently Vilas et al. (2006) reported aqueous alteration $(0.7 \mu \mathrm{m}$ band) on some satellites of the Jovian planets suggesting, suggesting that those bodies did not form in their present locations, but could have formed in the "aqueous alteration zone", from 2.6 - $3.5 \mu \mathrm{m}$, (Vilas et al. 1994) in the mainbelt. If the 0.6-0.65 $\mu \mathrm{m}$ and $0.8-0.9 \mu \mathrm{m}$ bands are due to aqueous alteration, their presence on D-types (main-belt, Trojans, and Chariklo) suggests that this kind of thermal processing on asteroids was not limited only to the $2.6-3.5 \mu \mathrm{m}$ zone, but happened in the whole main-belt, at least up to $5.2 \mathrm{AU}$ and in objects of the Centaur group.

\section{Acknowledgements}

This work has been supported by the Conselho Nacional de Desenvolvimento Científico e Tecnológico - CNPq/Brasil and Fundação de Amparo à Pesquisa do Estado do Rio de Janeiro - FAPERJ.

\section{References}

Abell, P. A., Fernández, Y. R., Pravec, P. et al. 2005, Icarus, 179, 174

A'Hearn, M. F., Campins, H., Schleicher, D. G., \& Millis, R. L. 1989, ApJ, 347, 1155

Burns, R. G. 1993, Mineralogical Applications of Crystal Field Theory, Cambridge Topics in Mineral Physics and Chemistry (Cambrigde: Cambridge University Press)

Bus, S. J. 1999, Compositional structure in the asteroid belt: results of a spectroscopic survey. $\mathrm{PhD}$ Dissertation. Massachusets Institute of Technology.

Bus, S. J. \& Binzel, R. P. 2002, Icarus, 158, 106

Barucci, M. A., Doressoundiram, A., Fulchignoni, M. et al. 1998, Icarus, 132, 388

Campins, H., A'Hearn, M. F., \& McFadden, L.-A. 1987, ApJ, 316, 847

Campins, H., Licandro, J., Ziffer, J. et al. 2005, IAU Symposium 229, abstracts book

Carvano, J. M., Mothé-Diniz, T., \& Lazzaro, D. 2003, Icarus, 161, 356

Cruikshank, D. P., Dalle Ore, C. M., Roush, T. L. et al. 2001, Icarus, 153, 348

Dahlgren, M., Lagerkvist, C. I., Fitzsimmons, A., Williams, I. P., \& Gordon, M. 1997, A\&A 323,606

Dotto, E., Fornasier, S., Barucci, M. A. et al. 2005, Icarus, submitted.

Dumas, C., Owen, T., \& Barucci, M. A. 1998, Icarus, 133, 221

Emery, J. P. \& Brown, R. H. 2003, Icarus 164, 104

Fitzsimmons, A., Dahlgren, M., Lagerkvist, C.-I., Magnusson, P., \& Williams, I.P. 1994, A\& $A$, 282,634

Fornasier, S., Dotto, E., Marzari, F. et al. 2004, Icarus, 172, 221

Harris, A. W., Delbó, M., Binzel, R. P. et al. 2001, Icarus, 153, 332

Jones T. D., Lebofsky L. A., Lewis J. S., \& Marley M. S. 1990, Icarus 88, 172

Jewitt D. C. \& Luu J. X. 1990, AJ, 100, 933

Kanno, A., Hiroi, T., Nakamura, R. et al. 2003, Geophys. Res. Letters, 30, 2

Lagerkvist, C.-I.,Fitzsimmons, A., Magnusson, P., \& Williams, I. P. 1993, MNRAS, 260, 679

Lagerkvist, C.-I., Moroz, L., Nathues, A. et al. 2005, A\&SA, 432, 349

Lazzaro, D., Angeli, C. A., Carvano, J. M. et al. 2004, Icarus, 172, 179

Lederer, S. M., \& Vilas, F. 2003, Earth Moon and Planets, 92, 193

Luu, J. X. 1993, Icarus, 104, 138

Millis, R. L., A’Hearn, M. F., \& Campins, H. 1988, ApJ, 324, 1194

Morbidelli, A., Levison, H. F., Tsiganis, K., \& Gomes, R. 2005, Nature, 435, 462 
Rivkin, A. S., Howell, E. S., Vilas, F., \& Lebofsky, L. A. 2003, in: W. F. Bottke Jr., A. Cellino, P. Paolicchi \& R. P. Binzel (eds.), Asteroids III, (Tucson: Univ. of Arizona), p. 235

Vilas, F. \& McFadden, L. A. 1992, Icarus, 100, 85

Vilas, F., Hatch, E. C., Larson, S. M., Sawyer, S. R., \& Gaffey, M. J. 1993, Icarus, 102, 225

Vilas F., Jarvis K. S., \& Gaffey, M. J. 1994, Icarus, 109, 274

Vilas F., Lederer S. M., Gillb S. L, Jarvisc K. S. \& Thomas-Osip, J.E. 2005, Icarus, 180, 453 